\title{
Statistical analysis plan for the POLAR-RCT: The Prophylactic hypOthermia trial to Lessen trAumatic bRain injury-Randomised Controlled Trial
}

Jeffrey Presneill ${ }^{1,6,21}$ BD, Dashiell Gantner ${ }^{1,2}$, Alistair Nichol ${ }^{1,2,3,4}$, Colin McArthur ${ }^{1,14}$, Andrew Forbes', Jessica Kasza', Tony Trapani 1,2, Lynnette Murray ${ }^{1}$, Stephen Bernard ${ }^{2,13}$, Peter Cameron ${ }^{5,7,19,20}$, Gilles Capellier ${ }^{15,16}$, Olivier Huet ${ }^{1,22,23}$, Lynette Newby $^{14}$, Stephen Rashford ${ }^{17}$, Jeffrey V. Rosenfeld ${ }^{10,12,18}$, Tony Smith ${ }^{8,14}$, Michael Stephenson ${ }^{1,13}$, Dinesh Varma ${ }^{9,10}$, Shirley Vallance ${ }^{1,2}$, Tony Walker ${ }^{13}$, Steve Webb ${ }^{1,11}$, D. James Cooper ${ }^{1,2^{*}}$ On behalf of the POLAR investigators and the ANZICS Clinical Trials Group

\begin{abstract}
Background: The Prophylactic hypOthermia to Lessen trAumatic bRain injury-Randomised Controlled Trial (POLAR-RCT) will evaluate whether early and sustained prophylactic hypothermia delivered to patients with severe traumatic brain injury improves patient-centred outcomes.

Methods: The POLAR-RCT is a multicentre, randomised, parallel group, phase III trial of early, prophylactic cooling in critically ill patients with severe traumatic brain injury, conducted in Australia, New Zealand, France, Switzerland, Saudi Arabia and Qatar. A total of 511 patients aged 18-60 years have been enrolled with severe acute traumatic brain injury. The trial intervention of early and sustained prophylactic hypothermia to $33^{\circ} \mathrm{C}$ for $72 \mathrm{~h}$ will be compared to standard normothermia maintained at a core temperature of $37^{\circ} \mathrm{C}$.

The primary outcome is the proportion of favourable neurological outcomes, comprising good recovery or moderate disability, observed at six months following randomisation utilising a midpoint dichotomisation of the Extended Glasgow Outcome Scale (GOSE). Secondary outcomes, also assessed at six months following randomisation, include the probability of an equal or greater GOSE level, mortality, the proportions of patients with haemorrhage or infection, as well as assessment of quality of life and health economic outcomes. The planned sample size will allow $80 \%$ power to detect a 30\% relative risk increase from 50\% to $65 \%$ (equivalent to a 15\% absolute risk increase) in favourable neurological outcome at a two-sided alpha of 0.05 .
\end{abstract}

Discussion: Consistent with international guidelines, a detailed and prospective analysis plan has been developed for the POLAR-RCT. This plan specifies the statistical models for evaluation of primary and secondary outcomes, as well as defining covariates for adjusted analyses and methods for exploratory analyses. Application of this statistical analysis plan to the forthcoming POLAR-RCT trial will facilitate unbiased analyses of these important clinical data.

Trial registration: ClinicalTrials.gov, NCT00987688 (first posted 1 October 2009); Australian New Zealand Clinical Trials Registry, ACTRN12609000764235. Registered on 3 September 2009.

Keywords: Traumatic brain injury, Cooling, Hypothermia, Outcome, Critical care, Randomised controlled trials

\footnotetext{
* Correspondence: Jamie.Cooper@monash.edu

${ }^{1}$ Australian and New Zealand Intensive Care Research Centre, Monash

University, School of Public Health and Preventive Medicine, 99 Commercial

Road, Melbourne 3004, Australia

${ }^{2}$ Department of Intensive Care, The Alfred, Melbourne, Australia

Full list of author information is available at the end of the article
} 


\section{Background}

Traumatic brain injury (TBI) is a leading cause of mortality and long-term disability, particularly affecting young people. Even a small increase in the number of TBI victims who are able to live independently, instead of being permanently disabled, would yield major human and economic benefits $[1,2]$.

The application of early prophylactic hypothermia [3] involves the rapid reduction after injury of core body temperature to $33{ }^{\circ} \mathrm{C}$. This therapy has shown promise as an intervention to attenuate TBI [4], with the distinctly early cooling intervention design of the Prophylactic hypOthermia to Lessen trAumatic bRain injury-Randomised Controlled Trial (POLAR-RCT; randomisation within $3 \mathrm{~h}$ of estimated time of injury) contrasting with a recent unsuccessful cooling intervention (therapeutic hypothermia) applied mostly $12 \mathrm{~h}$ or more after TBI $[5,6]$. POLAR-RCT is an international, multicentre, randomised, parallel group phase III superiority trial of prophylactic hypothermia delivered to adult critically ill patients with acute severe TBI (Glasgow Coma Score [GCS] <9) [7]. Despite a recent metaanalysis [8] suggesting improved neurological outcomes from TBI associated with therapeutic hypothermia to $33{ }^{\circ} \mathrm{C}$ for $72 \mathrm{~h}$, substantial clinical uncertainty remains in this field [9]. POLAR-RCT aims to evaluate six-month survival and neurological function in adult patients with severe TBI randomised to early prophylactic hypothermia to $33{ }^{\circ} \mathrm{C}$, compared to normothermia.

\section{Methods/Design}

\section{Study design and definitions}

The POLAR-RCT is a multicentre, prospective, two parallel groups, randomised phase III superiority trial evaluating the safety and efficacy of early prophylactic hypothermia targeting a core temperature of $33{ }^{\circ} \mathrm{C}$ in adult ICU patients with severe TBI using clinically available refrigerated vests, wraps, jackets, or blankets [7]. Control patients received protocol-directed temperature adjustment, using similar equipment if necessary, to maintain normothermia, defined as a core temperature of 36.5-37.5 ${ }^{\circ} \mathrm{C}$. Patients enrolled in both the hypothermia and normothermia groups have been managed according to current international evidence-based guidelines [10]. Patient randomisation completed on 10 November 2017 with a total of 511 participants and final collection of all six-month outcome data is anticipated by June 2018 .

\section{Treatment masking (blinding)}

Patient temperature is a key clinical vital sign and therefore it is not possible to blind clinical staff to treatment allocation. Bias will be minimised by concealed treatment allocation before randomisation, by protocolised treatment in both groups [7] and by assessment of the primary outcome by centralised, blinded and trained research staff (as accomplished successfully in recent studies including SAFE [11], SAFE-TBI [12], HTS [13] and ATBIS [2]). The primary outcome measure (neurological outcome at six months from randomisation, see below) is subject to minimal ascertainment bias.

\section{Compliance with good clinical practice}

The trial is being conducted, and accumulating data monitored, according to the standard requirements of Good Clinical Practice [14]. Data are collected by trained staff at each study site and entered into a secure, password-protected, encrypted web-based data collection form. Queries of potential inconsistencies are generated automatically by the trial website to facilitate early resolution. Data are stored in a secure server operated by Monash University; data management will be performed by the Clinical Informatics and Data Management Unit, Monash University, Melbourne, Australia [15]. Site monitoring will be performed at each participating hospital by the Trial Project Manager to ensure the study is conducted according to the protocol and all applicable regulations, and to perform source data verification (Additional file 1).

General confidence in the final results and conclusions of clinical trials is enhanced when the statistical approaches to outcome analyses are specified before the availability of trial data. The following statistical analysis plan complies with recommendations for the Consolidated Standards of Reporting Trials (CONSORT) (Fig. 1) $[16,17]$, the Standard Protocol Items: Recommendations for Interventional Trials (SPIRIT) (Fig. 2) [18, 19] (and checklist as an Additional file 2) as well as guidance from the International Conference on Harmonisation of Technical Requirements for Registration of Pharmaceuticals for Human Use, especially 'Statistical principles for clinical trials E9' [20] and 'Structure and content of clinical study reports E3' [21].

This statistical analysis plan identifies the procedures to be applied to the primary and secondary outcome analyses once trial data validation is complete. Covariates for adjusted analyses and selected subgroups of interest are also pre-specified. This plan defines the intention-to-treat (ITT) full analysis set, as well as exploratory analyses in 'as-treated' and 'per-protocol' subsets, accompanied by an analysis seeking to estimate the average causal effect of cooling in the presence of non-compliance with the cooling intervention [22].

\section{Trial population and eligibility}

A total of 511 severe TBI patients have been enrolled in Australia, New Zealand, France, Switzerland, Saudi Arabia and Qatar. This 511-patient full analysis set will 


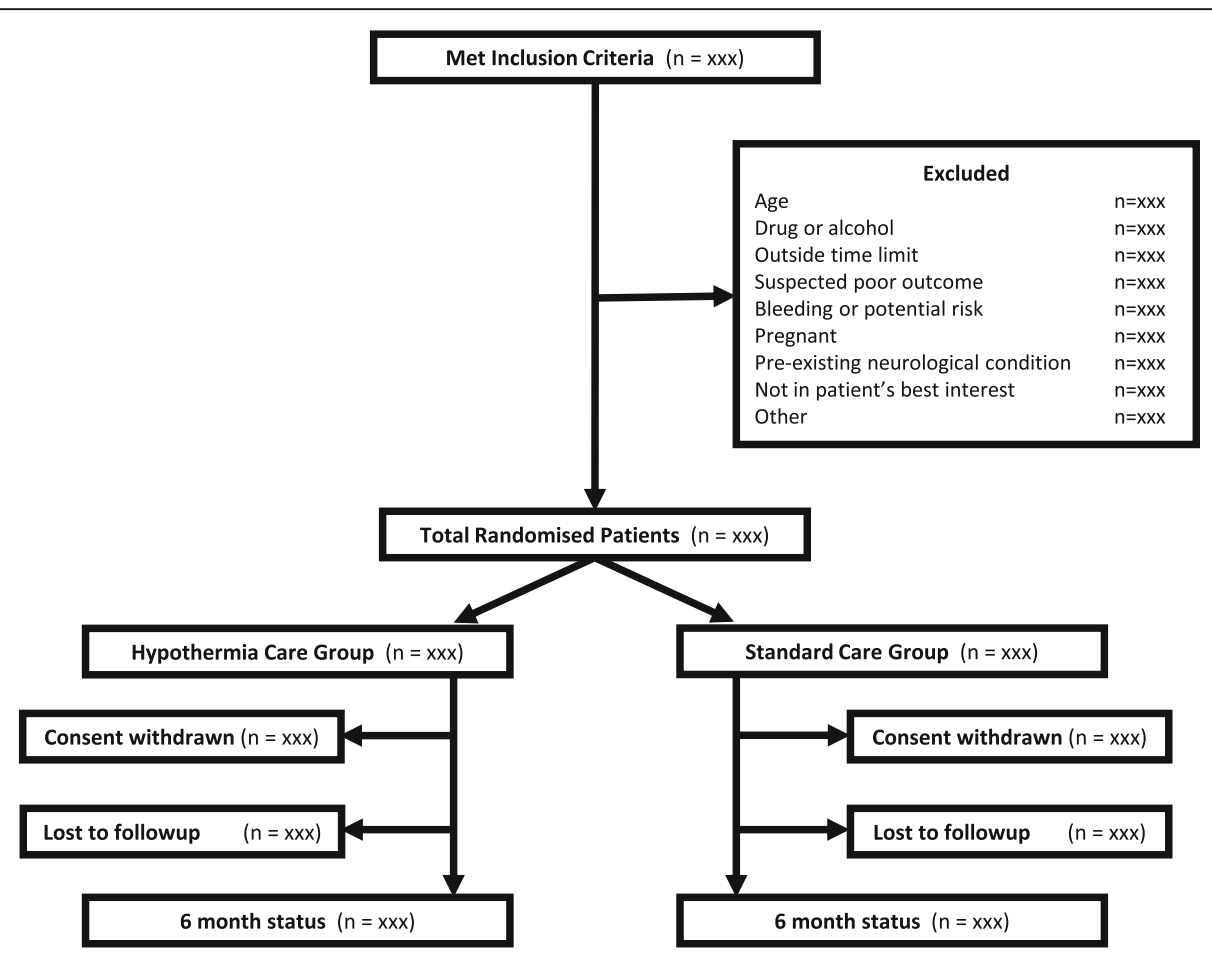

Fig. 1 Consort diagram

\begin{tabular}{|c|c|c|c|c|c|c|c|c|c|}
\hline \multirow[b]{3}{*}{ TIMEPOINT T* } & \multirow{3}{*}{$\begin{array}{c}\text { Enrolment } \\
0\end{array}$} & \multirow{3}{*}{\begin{tabular}{|c|} 
Allocation \\
0
\end{tabular}} & \multicolumn{7}{|c|}{ Study Period } \\
\hline & & & \multicolumn{6}{|c|}{ Post-allocation (Days) } & \multirow{2}{*}{$\begin{array}{c}\begin{array}{c}\text { Close-out } \\
\text { (Days) }\end{array} \\
180\end{array}$} \\
\hline & & & 1 & 2 & 3 & 4 & 10 & $\begin{array}{c}\text { Hospital } \\
\text { Discharge }\end{array}$ & \\
\hline \multicolumn{10}{|l|}{ ENROLMENT: } \\
\hline Eligibility screen & $\mathrm{X}$ & & & & & & & & \\
\hline Allocation & & $\mathrm{x}$ & & & & & & & \\
\hline \multicolumn{10}{|l|}{ Deferred consent } \\
\hline \multicolumn{10}{|l|}{ INTERVENTIONS: } \\
\hline \multicolumn{10}{|l|}{$\begin{array}{l}\text { Rapid cooling to } \\
33^{\circ} \mathrm{C}\end{array}$} \\
\hline \multicolumn{10}{|l|}{$\begin{array}{c}\text { Maintenance at } \\
33^{\circ} \mathrm{C}\end{array}$} \\
\hline \multicolumn{10}{|l|}{ Slow Rewarming } \\
\hline \multicolumn{10}{|l|}{$\begin{array}{c}\text { Standard Care } \\
\text { group }\end{array}$} \\
\hline \multicolumn{10}{|l|}{ ASSESSMENTS: } \\
\hline GCS / GCS (m) & $x$ & & & & & & & & \\
\hline Pupil reaction & $\mathbf{x}$ & & & & & & & & \\
\hline $\begin{array}{c}\text { Hypotension / } \\
\text { Hypoxia }\end{array}$ & $\mathbf{x}$ & & & & & & & & \\
\hline $\begin{array}{l}\text { MARSHALL } \\
\text { SCORE }\end{array}$ & & $x$ & & & & & & & \\
\hline CT SCAN & & $\mathrm{x}$ & & & & & & & \\
\hline Infection & & & & & & & $\mathrm{X}$ & & \\
\hline $\begin{array}{c}\text { Bleeding } \\
\text { (Intracranial) }\end{array}$ & & & & & & & $\mathrm{x}$ & & \\
\hline $\begin{array}{c}\text { Bleeding } \\
\text { (extracranial) }\end{array}$ & & & & & & & $x$ & & \\
\hline $\begin{array}{c}\text { Mortality Hospital } \\
\text { Discharge }\end{array}$ & & & & & & & & $x$ & \\
\hline $\begin{array}{c}\text { Mortality Hospital } \\
6 \text { month }\end{array}$ & & & & & & & & & $\mathbf{x}$ \\
\hline $\begin{array}{c}\text { GOSE / SF12/ } \\
\text { EQ5D3L }\end{array}$ & & & & & & & & & $\mathbf{x}$ \\
\hline
\end{tabular}

Fig. 2 SPIRIT figure 
not include the initial eight patients who were treated within a pre-trial run-in phase. Fourteen emergency departments and five pre-hospital agencies were involved. Patients may have been enrolled in the pre-hospital setting, if specifically trained and qualified staff are available, or by medical staff in the emergency department of participating hospitals. The inclusion and exclusion criteria are presented in Table 1. Eligible patients were randomised to receive prophylactic hypothermia $\left(33{ }^{\circ} \mathrm{C}\right.$ for $72 \mathrm{~h}$ ) or TBI care that is standard (for that hospital) (Figs. 1 and 2).

\section{Randomisation}

Randomisation 1:1 between trial hypothermia and normothermia was performed by paramedics or physicians using sequentially numbered, opaque, sealed envelopes (as used in the RICH [23] and RSI trials [24]), printed at the Australian trial coordinating office before distribution to sites. Envelopes were used because at the time of trial design it was not feasible to develop a centrally controlled, real-time electronic randomisation system across international emergency departments and pre-hospital ambulances services. The computer-generated randomised treatment allocation schedule (using Stata version 11 ralloc module http://fmwww.bc.edu/repec/bocode/r/ralloc.ado) [25] was stratified by hospital group or pre-hospital paramedic ambulance service with a permuted block scheme. Hospital strata were defined for participating hospitals in nine groups, comprising three Australian states (Victoria, Queensland, Western Australia), two localities in New Zealand (Auckland, Waikato) and four other countries (France, Switzerland, Saudi Arabia, Qatar). Stratification of pre-hospital randomisation schedules was also specified within the four networks where such enrolment was possible (Queensland, Victoria, Western Australia, France) (Table 2).

Patients who fulfilled the inclusion criteria and had no exclusion criteria were randomised by opening the next available opaque envelope within the ambulance or hospital emergency room.

In the pre-hospital setting patients were assessed by study trained and affiliated ambulance paramedics and/ or physicians. Patients who were enrolled in the study by a pre-hospital agency were assessed for study suitability and continuance on arrival in the first participating emergency department. Patients who were not enrolled in the study by a pre-hospital agency were assessed for study suitability on arrival in the associated hospital emergency department.

\section{Study objectives and endpoints Primary outcome}

The primary outcome will be each patient's neurological outcome at six months from randomisation. This is defined from a standard mid-point dichotomisation of the eight level ordinal Extended Glasgow

Table 1 Inclusion and exclusion criteria

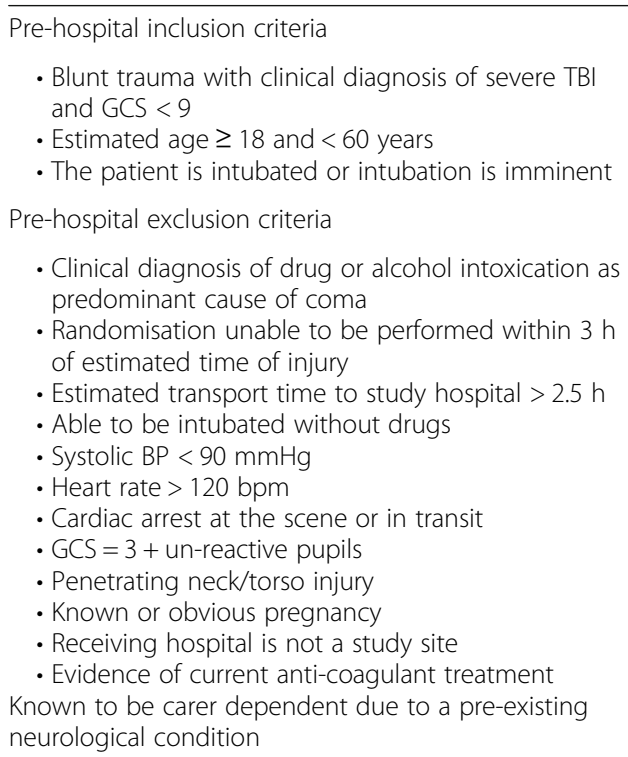

Emergency Department inclusion criteria

- Blunt trauma with clinical diagnosis of severe TB and GCS $<9$

- Estimated age $\geq 18$ and $<60$ years

- The patient is intubated or intubation is imminent

Emergency Department exclusion criteria

- Clinical diagnosis of drug or alcohol intoxication as predominant cause of coma

- Randomisation unable to be performed within $3 \mathrm{~h}$ of estimated time of injury

- Able to be intubated without drugs

- Persistent systolic BP $<90 \mathrm{mmHg}$

- GCS $=3+$ un-reactive pupils

- Cardiac arrest at the scene or in transit

- Clinically significant bleeding likely to require haemostatic intervention, for example:

- Bleeding into the chest, abdomen or retro-peritoneum likely to require surgery \pm embolisation

- Pelvic fracture likely to require surgery \pm embolisation

- More than two long bone fractures requiring operative fixation

- Penetrating neck/torso injury

- Positive urine or blood pregnancy test

- Evidence of current anti-coagulant treatment

- Known to be carer dependent due to a pre-existing neurological condition

- In the treating clinician's opinion, 'cooling' is not in the patient's best interest 
Table 2 The count of strata incorporated within the blockrandomised 1:1 hypothermia / normothermia treatment allocation schedule

\begin{tabular}{lcll}
\hline Pre-hospital & Stratum no. & Hospital region & Stratum no. \\
\hline Yes $^{\text {a }}$ & 1 & Victoria $^{\text {a }}$ & 5 \\
Yes $^{\text {a }}$ & 2 & Western Australia $^{a}$ & 6 \\
Yes $^{a}$ & 3 & Queensland $^{a}$ & 7 \\
Yes $^{a}$ & 4 & France $^{a}$ & 8 \\
No & & Switzerland & 9 \\
No & & Saudi Arabia & 10 \\
No & & Qatar & 11 \\
No & & Auckland & 12 \\
No & & Waikato & 13 \\
\hline
\end{tabular}

aparticipating pre-hospital ambulance services and their relevant regional hospitals within each of these Australia states and France were each included as separate strata within the computerised generation of randomisation envelopes

Outcome Scale (GOSE) [26] (Table 3), with favourable outcomes comprising lower moderate disability (GOSE 5) through upper good recovery (GOSE 8) and unfavourable outcomes comprising death (GOSE 1), the vegetative state (GOSE 2) or severe disability (GOSE 3 to 4).

\section{Secondary outcomes}

Secondary outcome measures are:

- GOSE regarded as an ordinal variable (as opposed to the dichotomisation used as the primary outcome variable)

- Quality of life (QOL) assessments at six months:

$$
\begin{aligned}
& \circ \text { EQ5D3L [27] } \\
& \circ \text { SF12 [28] }
\end{aligned}
$$

- Complier average causal effect of hypothermia on GOSE at six months comparing hypothermia and control patients

- Mortality (all cause) at:

$\circ$ hospital discharge

o six months

Table 3 The eight-level ordinal Extended Glasgow Outcome scale (GOSE)

\begin{tabular}{lll}
\hline Score & Description & Code \\
\hline 1 & Dead & D \\
2 & Vegetative state & VS \\
3 & Lower severe disability & SD - \\
4 & Upper severe disability & SD + \\
5 & Lower moderate disability & MD - \\
6 & Upper moderate disability & MD + \\
7 & Lower good recovery & GR - \\
8 & Upper good recovery & GR + \\
\hline
\end{tabular}

- Proportion of patients with adverse events (AEs) within ten days of randomisation, specifically:

o bleeding (intracranial, extracranial)

$\circ$ infection (by site)

- Cost-effectiveness at six months

\section{Statistical analyses}

Descriptive and summary statistics will be calculated by treatment group and stratum (pre-hospital and emergency department region) for baseline characteristics. Continuous data will be summarised as means (standard deviations) or medians (interquartile ranges) for nonnormal data and categorical data by counts and proportions. The number of screened patients who fulfilled study inclusion criteria and the number included in the primary and secondary analyses as well as all reasons for exclusions in primary and secondary analyses will be reported.

The main primary and secondary analyses will follow an ITT approach to define the full analysis patient set, based on all randomly assigned patients after the eightpatient run-in period, except those withdrawing consent for use of all trial data and those not fulfilling inclusion criteria and never receiving the intervention [20,29].

The early intervention design of the POLAR study specifically anticipates incomplete adherence to assigned cooling treatment in the complex clinical context of the study cohort. It is expected that the main reason for incomplete compliance with cooling will be due to the effects of alcohol or other drugs confounding assessment of TBI severity at the time of randomisation, leading to inclusion of patients with impaired consciousness not due to severe TBI for whom ongoing cooling may be clinically unwarranted. Other anticipated reasons include extracranial trauma and coincident haemorrhage for which cooling may be at least temporarily undesirable in the context of a patient's overall clinical condition.

\section{Primary outcome}

The primary outcome, the midpoint dichotomised GOSE, will be modelled as a binomial random variable, with a null hypothesis of equality between hypothermic and standard therapy groups in the proportion of subjects with an unfavourable outcome. This will be assessed with an uncorrected Chi-square test applied to the $2 \times 2$ contingency table comprising the full analysis set of patients according to randomised treatment group. This primary trial outcome will be reported as an unadjusted risk ratio with associated 95\% confidence interval (CI) and also as the risk difference with $95 \% \mathrm{CI}$ and odds ratio with 95\% CI. The number needed to treat for benefit or harm will also be reported if a statistically significant difference between treatment groups is demonstrated. 


\section{Secondary outcomes}

All secondary outcomes are hypothesis-generating. GOSE as an ordinal variable will be compared between treatment arms using a proportional odds model if the proportional odds assumption is justified [30,31]. If not, a partial proportional odds model will be used [32, 33].

Binary variables (including hospital and six-month mortality and AEs) will be analysed with log-binomial [34] and identity-binomial regression models [35] to estimate risk ratios and risk differences with $95 \%$ CIs, respectively.

QOL measures and other continuous variables will be analysed with linear regression, using robust standard errors to accommodate potential non-normality and unequal error variation.

Analyses of time-to-event outcomes will use KaplanMeier plots and log-rank tests, as well as unadjusted and adjusted Cox proportional hazard regression models returning hazard ratios with $95 \%$ CIs.

QOL outcomes will be reported as means (with standard deviations) of the physical and mental health scores of the SF-12 [28] and as the proportion of reported health problems for each domain of the EQ5D-3 L [27]. Differences in QOL between groups will be assessed using two-sample $t$ tests or Wilcoxon rank-sum tests as appropriate [20] for the SF-12 component scores and Fisher's exact or Chi-squared tests for the proportions in each domain of the EQ-5D-3 L.

\section{Cost-effectiveness analyses}

Cost-effectiveness from the healthcare-payer perspective will be calculated as a cost per additional patient with a favourable neurological outcome at six months following randomisation (defined as GOSE 5-8) and the cost per additional quality-adjusted life year (QALY), with QALYs calculated using utility scores derived from the EQ-5D$3 \mathrm{~L}$ conducted at six months post randomisation. Costs will be determined based on resource use during the intensive care, acute and post-acute periods up to six months post randomisation. These will be valued using the UK time-trade-off tariff. All patients will be assumed to have a utility score of zero at randomisation in accordance with other studies recruiting patients in critical care. Patients who die before the six-month follow-up will be given a utility of zero.

Data on resource use at different levels of care will be recorded at discharge from the index hospitalisation and again at follow-up for subsequent healthcare resource use (including readmissions, rehabilitation and other care facilities). Information will be collected for length of ventilation, time in ICU, hospital (ward) days, rehabilitation days and time spent in high- and low-level care facilities and transitional living centres.
Resources will be translated into costs by multiplying the relevant country-specific unit cost by use for each patient in the analysis, where unit costs are obtained from hospital staff where possible, or national databases. All costs will be reported in 2017 prices using national consumer price index statistics from relevant countries. Purchasing power parity (PPP) statistics from the Organisation for Economic Co-operation and Development (OECD) will be used to translate costs to a common currency (United States Dollars, \$US). Given the relatively short time period, costs will not be discounted.

Analyses will adjust for fixed effects across three geographical regions (Australia/New Zealand (ANZ), Europe and the Middle East) to account for potential heterogeneity which may arise, for example, through regional variations in treatment patterns. The issue of transferability in multinational trials will be addressed by estimating costs and effects for the same three geographical regions. Region-specific estimates will be obtained by interacting the treatment variable with regional fixed effects.

\section{Pre-specified subgroup analyses}

Two interactions of particular prior interest will be assessed. The primary and secondary outcomes will be evaluated according to: (1) the presence of surgically evacuated intracranial mass lesions (Marshall score V) [36]; and (2) the presence of any intracranial mass lesion whether or not surgically evacuated (Marshall V or VI).

\section{Dose effect / Intensity of cooling}

Intensity of cooling in intervention arm patients will be categorised according to the time after randomisation to first reach one of two core temperature thresholds, being the limit of mild hypothermia $35{ }^{\circ} \mathrm{C}[9]$ and also $34{ }^{\circ} \mathrm{C}$ as an indicator of more intense [6] cooling below $35{ }^{\circ} \mathrm{C}$ towards $33{ }^{\circ} \mathrm{C}$. Cooling intensity categories are defined as never achieving hypothermia and tertiles of time in those reaching hypothermia. Primary and secondary outcomes of patients in these intensity categories will be compared across categories and to standard care patients.

Differential effects of cooling on the primary and secondary outcomes will be assessed according to: (1) Marshall computed tomography (CT) scan classification $\mathrm{V}$; and (2) Marshall V or VI. These will be performed using appropriate covariate by treatment interaction terms in the relevant regression models.

Sensitivity analyses of the primary and secondary outcomes Sensitivity analyses of the primary and secondary outcomes will be performed using regression models adjusting for pre-specified baseline covariates as well as any covariate exhibiting substantial imbalance between randomisation arms, as recommended [37]. 
Linear and generalised linear model diagnostics, outlier assessment and remedial measures will follow standard approaches [30, 38].

Proportionality in ordinal logistic regression models will be assessed [39]. Also, the proportional hazards assumption across treatment arms in time-to-event analyses will be evaluated using scaled Schoenfeld residuals [40] and visual assessment of log-log plots.

Baseline variables to be included as fixed effects when developing adjusted outcomes models comprise the following:

- Geographic region (Australia and New Zealand, Middle East, Europe - three level nominal categorical)

- Age (integer values centred on overall median age of trial patients)

- GCS (integer values with a possible range of 3-15)

- Pupil reactivity (ordinal, three levels)

- Hypoxia (binary)

- Hypotension (binary)

- Marshall CT brain scan classification (nominal categorical, six levels) [39]

- Core temperature closest to time of randomisation (continuous)

Generalised linear mixed regression models will be applied with the same fixed effects covariate set while also incorporating as a random effect the multiple randomisation strata comprising hospital and pre-hospital research groups. In the event the above regression models do not accommodate the number of prespecified covariates, sensitivity analyses will be simplified to adjust at least for POLAR treatment and the baseline extended IMPACT TBI probability of 6 month unfavourable outcome.

\section{Exploratory analyses}

It is well understood that trial protocols may not have been followed fully for some trial participants [17]. The intervention under examination is hypothermia to a target of $33{ }^{\circ} \mathrm{C}$ core temperature initiated within $3 \mathrm{~h}$ of injury and continued for at least $72 \mathrm{~h}$, with rewarming guided by an intracranial pressure $<20 \mathrm{mmHg}$. Beyond the main ITT analyses of the primary and secondary outcomes, pre-specified exploratory analyses will use 'astreated' and 'per-protocol' comparison approaches as well as methods to estimate the complier average causal effect' (CACE) of cooling [22, 38].

As-treated and per-protocol analyses POLAR will repeat the primary and secondary trial outcomes in two pre-defined exploratory analysis sets: (1) the subset of compliant patients assigned to cooling compared to all patients assigned to the control ('per-protocol' analysis); and (2) according to the actual treatment received ('astreated' analysis) [7].

The per-protocol analysis subset will comprise control and intervention patients who are demonstrated retrospectively to have suffered a severe TBI, thus excluding those with initially low GCS $<9$ not due to severe head injury but rather associated with transient influences, such as alcohol or drug effects.

For analytic purposes, the differential 'dose' of early cooling received by each patient will be summarised as the area (degree $\times$ hours) under the protocol-defined cooling baseline value of $35{ }^{\circ} \mathrm{C}$ within $72 \mathrm{~h}$ of randomisation. Each individual's AUC will be estimated by the linear trapezoidal method [41] applied to temperature data ignoring missing values. Early death or loss to follow-up within $72 \mathrm{~h}$ of randomisation will by definition reduce the observed dose of cooling received by such patients. The intensity of cooling will also be summarised categorically, in increments of $1{ }^{\circ} \mathrm{C}$ from $\geq 38^{\circ} \mathrm{C}$ to $\leq 32{ }^{\circ} \mathrm{C}$, as the proportion of individuals whose lowest core temperature during the trial intervention days was in each of these temperature bands. A binary definition of 'compliance with cooling' will constitute an AUC at least 72 degree $\times$ hours, this being at least half that theoretically achievable in the first $72 \mathrm{~h}$. This definition will be used to identify the per-protocol and as-treated populations.

Estimation of the average causal effect among patients complying with treatment In trials with incomplete adherence to treatment, such as that anticipated in POLAR, the ITT comparison only provides an estimate of the causal effect of treatment assignment rather than an estimate of the causal effect of treatment actually received. CACE analyses will be conducted to estimate the average effect of cooling treatment on the primary outcome for patients who would comply with whichever cooling group they were assigned to, considering both the binary and continuous definitions of compliance with cooling [22, 38].

Estimation of the CACE will use an instrumental variables approach in the first instance, with a randomised arm as the instrument [42]. Alternatively, if compliance is a time-varying variable, estimation of the CACE will use longitudinal nested compliance class methods [43].

\section{Data monitoring}

All numerical data fields in the trial database have upper and lower review limits based on biologically unlikely thresholds, so as to identify possible data entry errors. Also, all dates and times will be checked for logic errors. The POLAR Trial Manager monitored all research sites in person, with the assistance of a French-speaking trial 
monitor at the four sites in France. Monitoring visits checked multiple aspects of data validity, including consent documents, inclusion and exclusion criteria, AEs and important daily data such as temperature, use of vasoactive agents, fluid balance, blood transfusion and electrolyte disturbances (Additional file 3).

An independent Data and Safety Monitoring Committee (DSMC) continues to oversee the quality of the trial and has access to trial outcome and accumulated safety data, including the differential proportions of total mortality (Additional file 4).

\section{Sample size, power and interim analysis schedule}

The overall recruitment target was set at 500 participants from commencement in December 2010 and was slightly increased to 510 participants in September 2017 after blinded review of the combined proportion of patients with 'consent withdrawn' or 'loss to follow-up'. A total of 511 patients were randomised before the conclusion of trial recruitment on 10 November 2017.

A study of fixed size with full compliance and followup would require 364 patients (182 in each of two treatment arms) to detect a 15\% absolute increase from 50\% to $65 \%$ in favourable neurological outcome at six months following injury (equivalent to a $30 \%$ relative improvement in risk) with slightly $>80 \%(82.8 \%)$ power, assuming a two-sided alpha of 0.05 and an uncorrected Chi square test. This postulated POLAR risk improvement from a baseline of $50 \%$ to $65 \%$ in treated patients is equivalent to an odds ratio of approximately 1.86.

The original POLAR trial sample size of 364 fully evaluable patients was appropriately inflated to a practically required target of 500 patients to maintain $80 \%$ power to find the anticipated beneficial effect of prophylactic hypothermia while accommodating anticipated losses to follow-up (5\%) and non-compliance (cross-over from cooling to control and related losses, maximum 12\%). Also incorporated was a much smaller $(0.7 \%)$ inflation necessary to accommodate the originally anticipated interim analyses of both mortality and the proportion of unfavourable neurological outcomes using HaybittlePeto 3SD group sequential boundaries at two recruitment points (one-quarter $[n=125]$ and one-half [n=250]) [7].

Following the October 2015 publication of the EUROTHERM3235 study [6], the POLAR DSMC required a further substantial increase in interim monitoring, namely at increments of 50 patients from $n=300$ to $n=450$ inclusive. Ten of 11 planned interim analyses will be of both mortality and the proportion of unfavourable neurological outcomes, while at the penultimate $(n=450)$ assessment, short-term 28-day mortality alone will be assessed due to time constraints. These extra analyses implied a sample size inflationary requirement $(4 \%-0.7 \%=3.3 \%$ extra $)$ [44] which may be accommodated within the originally planned sample size, provided losses to follow-up and non-compliance remained below anticipated limits.

From calculations using East trial design software [45] based upon an approximate Chi-square test, the trial power was only slightly diminished at $82.3 \%$ (down from 83\%) with 366 fully evaluable participants and 11 interim Haybittle-Peto 3SD interim analyses. In the absence of early stopping, the final analysis would be properly conducted at $\pm 1.996 \mathrm{SD}(P=0.0459)$ rather than $\pm 1.96 \mathrm{SD}$ $(P=0.05)$. This level of significance will not be adjusted for multiplicity; however, the primary trial outcome is clearly defined and the conclusions of the study will be those based on the primary analysis conducted in the ITT full analysis patient set. Unless otherwise specified, all hypothesis tests and accompanying significance levels (that is, $P$ values) will be two-sided, with $95 \% \mathrm{CI}$.

\section{Analysis software}

Data capture and processing occurs initially at Monash University Clinical Informatics and Data Management Unit [15] and these data will be exported in relevant formats for statistical analysis using current versions of SAS software [46] and Stata software [47] or similar statistical software.

\section{Safety and adverse event analyses}

Safety and tolerability implications will be summarised using descriptive statistical methods, supplemented by calculation of $95 \%$ CI where appropriate. Patients with protocol deviations, AEs (Additional file 5: POLAR Data Dictionary Form12 Adverse Events) and missing values will be identified, and a descriptive analysis undertaken including their relationship to treatment.

\section{Discussion}

Severe TBI is a common and devastating condition with few proven specific therapies available. The administration of early prophylactic hypothermia has the potential to reduce neurological damage and improve neurological outcome and is supported by a scientific rationale and laboratory data. The POLAR-RCT is designed to detect an important beneficial effect of early therapeutic hypothermia on neurological function if one exists, while minimising any potential risks after TBI with accompanying illness or extracranial injury. Application of this statistical analysis plan to the POLAR-RCT trial will facilitate evaluation of these important clinical data and support confidence in the subsequent generalisation of its findings. POLAR-RCT aims to provide definitive guidance for clinicians regarding the true efficacy and safety of early prophylactic hypothermia in the management of TBI. 


\section{Trial status}

The POLAR-RCT began in December 2010, with patient randomisation completed on 10 November 2017 and final collection of all six-month outcome data anticipated by June 2018. A published manuscript describes the details of the trial protocol [7]. The current protocol is version 9, dated 11 July 2017.

\section{Additional files}

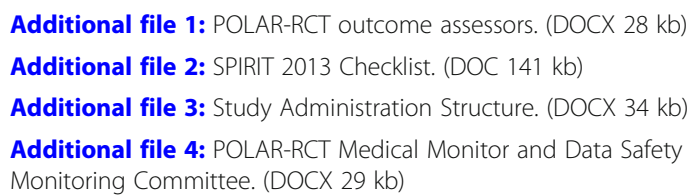

Additional file 5: POLAR Data Dictionary Form12 Adverse Events. (DOCX $45 \mathrm{~kb}$ )

Additional file 6: POLAR-RCT Management Committee and Coordinating Centre. (DOCX 29 kb)

Additional file 7: The POLAR Study Investigators. (DOCX $31 \mathrm{~kb}$ )

Additional file 8: Responsible Ethics Committees. (DOCX 30 kb)

\section{Abbreviations}

ANZIC-RC: Australian and New Zealand Intensive Care Research Centre; AUC: Area under curve; Cl: Confidence interval; DSMC: Data and Safety Monitoring Committee; EQ-5D: EuroQol five dimensions; GCS: Glasgow Coma Score; GOSE: Extended Glasgow Outcome Scale; SF-12: Short Form-12; TBI: Traumatic brain injury

\section{Acknowledgements}

Professor John Myburgh contributed to the initial successful grant application and Initial Protocol development.

\section{Funding}

Funding for this investigator-initiated research is sourced in Australia from the National Health and Medical Research Council (Project Grant ID 545901) and the Victorian Transport Accident Commission (formerly Victorian Neurotrauma Initiative; Project Grant ID D162). Trial design, data collection and analyses will be undertaken independent of the funding bodies. The POLAR-RCT trial is co-ordinated by the Australian and New Zealand Intensive Care-Research Centre, Monash University, Melbourne and endorsed by the Australian and New Zealand Intensive Care Society Clinical Trials Group. POLAR-RCT is managed in France by the Centre Hospitalier Régional Universitaire (CHRU) Besançon. The members of the Management Committee are listed in Additional file 6. The POLAR-RCT trial sites, principal investigator(s) and research coordinator(s) are listed in Additional file 7.

\section{Availability of data and materials}

The datasets to be generated and/or analysed during the current study are not publicly available as the trial database is not yet locked and analyses have not yet commenced. In the future, data will be available from the corresponding author on reasonable request.

\section{Authors' contributions}

Successful grant applications and Initial Protocol development of POLAR was performed by DJC, SB, AN, JP, PC, AF, SW, JR, LM, CM, LN, TS and TW. Contributions to subsequent POLAR Protocol Versions were made by DJC, SB, AN, JP, PC, AF, SW, JR, LM, CM, LN, TS, TW, TT, SR, MS, MB, SV, GC and DV. Development and publication of the POLAR Protocol manuscript which supported this subsequent statistical analysis plan involved DJC, DG, SB, AN JP, PC, AF, SW, JR, LM, CM, LN, TS, TW, TT, SR, MS, GC and DV. All authors have been involved with development or review of the current POLAR Statistical analysis plan manuscript including DJC, SB, AN, JP, PC, AF, SW, JR, LM, CM, LN, TS, TW, TT, SR, MS, GC, DV, DG, SV, OH and JK. All authors read and approved the final manuscript.

\section{Ethics approval and consent to participate}

Ethics and regulatory approvals of the protocol and related documents were obtained before commencing the trial at each site according to state or national legislation. The list of responsible ethics committees is provided in Additional file 8. The study protocol has been updated on several occasions, with each amendment receiving further ethics and regulatory approval. Version 9 is current from 11 July 2017.

Unconscious patients with severe TBI will not be able to provide informed consent; therefore, this trial uses a deferral of consent procedure. Informed consent will be obtained from each patient's legal surrogate for data collection as soon as reasonably possible and appropriate after injury. Patients who recover sufficient cognition to understand an explanation of the trial will additionally be asked to consent to continuation in the trial and the use of their data in the trial, if this is required under the ethics committee approval conditions. In France, patients may be enrolled under an 'Emergency clause'. More details of the trial protocol and participating hospitals are available from the trial registration site [48] as well as from the published study protocol [7].

\section{Competing interests}

The authors declare that they have no competing interests.

\section{Publisher's Note}

Springer Nature remains neutral with regard to jurisdictional claims in published maps and institutional affiliations.

\section{Author details}

${ }^{1}$ Australian and New Zealand Intensive Care Research Centre, Monash University, School of Public Health and Preventive Medicine, 99 Commercial Road, Melbourne 3004, Australia. ${ }^{2}$ Department of Intensive Care, The Alfred, Melbourne, Australia. ${ }^{3}$ Department of Anaesthesia and Intensive Care Medicine, St Vincent's University Hospital, Dublin, Ireland. ${ }^{4}$ School of Medicine and Medical Sciences, University College, Dublin, Ireland. ${ }^{5}$ Centre of Excellence in Traumatic Brain Injury Research, The Alfred, Monash University, Melbourne, Australia. ${ }^{6}$ Intensive Care Unit, Royal Melbourne Hospital, Melbourne, Australia. ${ }^{7}$ Emergency Medicine, Hamad Medical Corporation, Doha, Qatar. ${ }^{8}$ St John New Zealand, Auckland, New Zealand. ${ }^{9}$ Department of Radiology, The Alfred Hospital, Melbourne, Australia. ${ }^{10}$ Department of Surgery, Monash University, Melbourne, Australia. ${ }^{11}$ Intensive Care Unit, Royal Perth Hospital, Perth, Australia. ${ }^{12}$ Department of Neurosurgery, The Alfred Hospital, Melbourne, Australia. ${ }^{13}$ Ambulance Victoria, Melbourne, Australia. ${ }^{14}$ Department of Critical Care Medicine, Auckland City Hospital, Auckland, New Zealand. ${ }^{15}$ Réanimation médicale CHRU Jean Minjoz, Besançon, France. ${ }^{16}$ Université de Franche - Comte, 1 Rue Claude Goudimel, Besançon 25030, France. ${ }^{17}$ Queensland Ambulance Service, Brisbane, Australia. ${ }^{18}$ Department of Surgery, F. Edward Hébert School of Medicine, Uniformed Services University of The Health Sciences (USUHS), Bethesda, MD, USA. ${ }^{19}$ Emergency and Trauma Centre, The Alfred Hospital, Melbourne, Australia. ${ }^{20}$ School of Public Health and Preventive Medicine, Monash University, Melbourne, Australia. ${ }^{21}$ Department of Medicine, University of Melbourne, Melbourne, Australia. ${ }^{22}$ Department of Anaesthesia and Intensive Care Medicine, Hôpital de La Cavale Blanche, CHRU de Brest, Brest, France. ${ }^{23}$ UFR de médecine et des sciences de la santé, Université de Bretagne Occidental, Brest, France.

Received: 12 May 2017 Accepted: 27 March 2018

Published online: 27 April 2018

\section{References}

1. Nichol AD, Higgins AM, Gabbe BJ, Murray $\amalg$, Cooper DJ, Cameron PA. Measuring functional and quality of life outcomes following major head injury: common scales and checklists. Injury. 2011;42:281-7.

2. Myburgh JA, Cooper DJ, Finfer SR, Venkatesh B, Jones D, Higgins A, et al. Epidemiology and 12-month outcomes from traumatic brain injury in Australia and New Zealand. J Trauma. 2008;64:854-62.

3. Bernard SA, Buist M. Induced hypothermia in critical care medicine: a review. Crit Care Med. 2003;31:2041-51.

4. Nichol AD, Trapani T, Murray L, Vallance S, Cooper DJ. Hypothermia in patients with brain injury: the way forward? Lancet Neurol. 2011;10:405-7.

5. Cooper DJ, Nichol A, Presneill J. Hypothermia for intracranial hypertension after traumatic brain injury. N Engl J Med. 2016;374:1384. 
6. Andrews PJ, Sinclair HL, Rodriguez A, Harris BA, Battison CG, Rhodes JK, et al. Hypothermia for intracranial hypertension after traumatic brain injury. N Engl J Med. 2015;373:2403-12.

7. Nichol A, Gantner D, Presneill J, Murray L, Trapani T, Bernard S, et al. Protocol for a multicentre randomised controlled trial of early and sustained prophylactic hypothermia in the management of traumatic brain injury. Crit Care Resusc. 2015;17:92-100

8. Crompton EM, Lubomirova I, Cotlarciuc I, Han TS, Sharma SD, Sharma P. Meta-analysis of therapeutic hypothermia for traumatic brain injury in adult and pediatric patients. Crit Care Med. 2017:45:575-83.

9. Lewis SR, Evans DJ, Butler AR, Schofield-Robinson OJ, Alderson P. Hypothermia for traumatic brain injury. Cochrane Database Syst Rev. 2017;9:CD001048. https:/doi.org/10.1002/14651858.CD14001048.pub14651855

10. Brain Trauma Foundation. Guidelines for the management of severe traumatic brain injury. J Neurotrauma. 2007:24(Suppl 1):S1-106.

11. Finfer S, Bellomo R, Boyce N, French J, Myburgh J, Norton R, et al. A comparison of albumin and saline for fluid resuscitation in the intensive care unit. N Engl J Med. 2004;350:2247-56.

12. The Safe Study Investigators. Saline or albumin for fluid resuscitation in patients with traumatic brain injury. N Engl J Med. 2007;357:874-84.

13. Cooper DJ, Myles PS, McDermott FT, Murray LJ, Laidlaw J, Cooper G, et al. Prehospital hypertonic saline resuscitation of patients with hypotension and severe traumatic brain injury: a randomized controlled trial. J Am Med Assoc. 2004;291:1350-7.

14. International conference on harmonisation E6(R1); Guideline for good clinical practice. http://www.ich.org/fileadmin/Public_Web_Site/ICH_ Products/Guidelines/Efficacy/E6/E6_R1_Guideline.pdf. Accessed 17 Apr 2017

15. Monash University Clinical Informatics and Data Management Unit (CIDMU). https://www.monash.edu/medicine/sphpm/depts-centres-units/cidmu. Accessed 17 Apr 2017

16. Schulz KF, Altman DG, Moher D. CONSORT 2010 statement: updated guidelines for reporting parallel group randomised trials. Br Med J. 2010;340:c332.

17. Moher D, Hopewell S, Schulz KF, Montori V, Gotzsche PC, Devereaux PJ, et al. CONSORT 2010 explanation and elaboration: updated guidelines for reporting parallel group randomised trials. Br Med J. 2010;340:C869.

18. Chan A-W, Tetzlaff JM, Gøtzsche PC, Altman DG, Mann H, Berlin JA, et al, SPIRIT 2013 explanation and elaboration: guidance for protocols of clinical trials. BMJ. 2013;346:e7586.

19. Chan A, Tetzlaff JM, Altman DG, Laupacis A, Gotzsche PC, Krleza-Jeric K, et al. Spirit 2013 statement: Defining standard protocol items for clinical trials. Ann Intern Med. 2013;158:200-7.

20. International conference on harmonisation E9; statistical principles for clinical trials. http://www.ich.org/fileadmin/Public_Web_Site/ICH_Products/ Guidelines/Efficacy/E9/Step4/E9_Guideline.pdf. Accessed 17 Apr 2017.

21. International conference on harmonisation E3; Structure and content of clinical study reports. http://www.ich.org/fileadmin/Public_Web_Site/ICH_ Products/Guidelines/Efficacy/E3/E3_Guideline.pdf. Accessed 17 Apr 2017.

22. Imbens GW, Rubin DB. Causal inference for statistics, social, and biomedical sciences: An introduction. 1st ed. New York: Cambridge University Press; 2015

23. Bernard SA, Smith K, Cameron P, Masci K, Taylor DM, Cooper D, et al. Induction of prehospital therapeutic hypothermia after resuscitation from nonventricular fibrillation cardiac arrest. Crit Care Med. 2012;40:747-53.

24. Bernard SA, Nguyen V, Cameron P, Masci K, Fitzgerald M, Cooper DJ, et al. Prehospital rapid sequence intubation improves functional outcome for patients with severe traumatic brain injury: a randomized controlled trial. Ann Surg. 2010;252:959-65.

25. Stata Corporation. 2010. Stata: Release 11.1. www.stata.com.

26. Teasdale GM, Pettigrew LE, Wilson JT, Murray G, Jennett B. Analyzing outcome of treatment of severe head injury: a review and update on advancing the use of the Glasgow Outcome Scale. J Neurotrauma. 1998;15:587-97.

27. EQ-5D-3L a standardized non-disease specific instrument to describe and value health-related quality of life. https://euroqol.org/eq-5d-instruments/ eq-5d-3l-about/. Accessed 06 Nov 2017

28. Ware J Jr, Kosinski M, Keller SD. A 12-Item Short-Form Health Survey: construction of scales and preliminary tests of reliability and validity. Med Care. 1996;34:220-33.

29. Fergusson D, Aaron SD, Guyatt G, Hebert P. Post-randomisation exclusions: the intention to treat principle and excluding patients from analysis. Br Med J. 2002;325:652-4.
30. Hosmer DW, Lemeshow S, Sturdivant RX. Applied logistic regression. Hoboken: Wiley; 2013.

31. Roozenbeek B, Lingsma HF, Perel P, Edwards P, Roberts I, Murray GD, et al. The added value of ordinal analysis in clinical trials: an example in traumatic brain injury. Crit Care. 2011;15:R127.

32. Ananth CV, Kleinbaum DG. Regression models for ordinal responses: a review of methods and applications. Int J Epidemiol. 1997;26:1323-33.

33. Peterson B, Harrell FE Jr. Partial proportional odds models for ordinal response variables. J R Stat Soc: Ser C: Appl Stat. 1990;39:205-17.

34. Marschner IC, Gillett AC. Relative risk regression: reliable and flexible methods for log-binomial models. Biostatistics. 2012;13:179-92.

35. Kovalchik SA, Varadhan R, Fetterman B, Poitras NE, Wacholder S, Katki HA. A general binomial regression model to estimate standardized risk differences from binary response data. Stat Med. 2013;32:808-21.

36. Marshall LF, Marshall SB, Klauber MR, Van Berkum CM, Eisenberg H, Jane JA et al. The diagnosis of head injury requires a classification based on computed axial tomography. J Neurotrauma. 1992;9(Suppl 1):S287-92.

37. Guideline on adjustment for baseline covariates. http://www.ema.europa.eu/ docs/en_GB/document_library/Scientific_guideline/2015/03/WC500184923. pdf. Accessed 17 Apr 2017.

38. Vittinghoff E, Glidden DV, Shiboski SC, McCulloch CE. Regression methods in biostatistics: linear, logistic, survival, and repeated measures models. In: Statistics for biology and health. 2nd ed. New York: Springer-Verlag; 2012. p. XX, 512.

39. Brant R. Assessing proportionality in the proportional odds model for ordinal logistic regression. Biometrics. 1990;46:1171-8.

40. Grambsch PM, Therneau TM. Proportional hazards tests and diagnostics based on weighted residuals. Biometrika. 1994;81:515-26.

41. Spritzler J, DeGruttola VG, Pei L. Two-sample tests of area-under-the-curve in the presence of missing data. Int J Biostat. 2008;4:Article 1.

42. Hernan MA, Robins JM. Per-protocol analyses of pragmatic trials. N Engl J Med. 2017:377:1391-8.

43. Lin JY, Have TRT, Elliott MR. Longitudinal nested compliance class model in the presence of time-varying noncompliance. J Am Stat Assoc. 2008;103:462-73.

44. Jennison C, Turnbull BW. Group sequential methods with applications to clinical trials. Boca Raton: Chapman \& Hall/CRC; 2000.

45. Cytel Inc. 2016. East 6.4. www.cytel.com

46. SAS Institute Inc. 2017. SAS software. https://www.sas.com/en_au/ software/sas9.html.

47. Stata Corporation. 2016. Stata Statistical Software: Release 14.2. http:// www.stata.com/.

48. The Prophylactic Hypothermia Trial to Lessen Traumatic Brain Injury (POLARRCT). https://clinicaltrials.gov/ct2/show/NCT00987688?term=polar+rct\&rank=1. Accessed 17 Apr 2017

\section{Submit your next manuscript to BioMed Central and we will help you at every step:}

- We accept pre-submission inquiries

- Our selector tool helps you to find the most relevant journal

- We provide round the clock customer support

- Convenient online submission

- Thorough peer review

- Inclusion in PubMed and all major indexing services

- Maximum visibility for your research

Submit your manuscript at www.biomedcentral.com/submit
) Biomed Central 